\title{
Dos dispositivos móveis à aprendizagem ubíqua - Da usabilidade técnica à usabilidade pedagógica
}

\author{
Viviane Gomes da Silva*, Maria João Gomes**, \\ * Instituto Federal de Educação Ciência e Tecnologia do Amazonas - Brasil, Doutoranda em Ciências da Educação, especialidade em \\ Tecnologia Educativa pela Universidade do Minho, ** Professora Assistente do Instituto de Educação da Universidade do Minho
}

\begin{abstract}
Resumo
$\mathrm{Na}$ sociedade digital, as aprendizagens -móvel e ubíqua- tornaram-se notáveis no cenário educativo. O que ampliou a aprendizagem nomeadamente do espaço físico para o ciberespaço. Este artigo apresenta o projeto de pesquisa de doutoramento em Tecnologias Educativas em Ciências da Educação aprovado no comitê científico do IE da Universidade do Minho em Maio de 2015. Tese em andamento cujo objetivo é articular os conceitos de Usabilidade Técnica e Usabilidade Pedagógica aplicados no âmbito da aprendizagem móvel e ubíqua. Portanto, não há conclusões ou considerações finais, posto que o estágio situa-se em fase inicial.
\end{abstract}

Palavras-chaves: Aprendizagem Ubíqua, Aprendizagem Móvel, Usabilidade, Usabilidade Pedagógica.

\section{Introdução}

A sociedade encontra-se na era da mobilidade e do ciberespaço, ou seja, desterritorializada. A possibilidade de permanecer conectado sob as formas tecnológicas atuais de comunicação sem fio representa novas práticas e comportamentos de comunicação e partilha de informações no ciberespaço (Lemos, 2007).

As mudanças no mundo científico, tecnológico, cultural, social e educacional ocorridas foram determinantes para aproximar os benefícios da tecnologia às necessidades da educação. No bojo destas transformações as tecnologias móveis e ubíquas transformam a forma de interagir com a sociedade, desde o relacionamento entre as pessoas, os novos postos de empregos, os serviços disponíveis além da escolha na formação ou qualificação profissional.

Neste cenário as Instituições de Ensino Superior (IES) debruçam-se sob novas perspectivas de contribuir na transformação da sociedade na era do conhecimento. Diante deste contexto, tem adquirido importância crescente a educação a distância $(\mathrm{EaD})$, modalidade de ensino que permite a expansão do ensino por meio da utilização de Tecnologias de Informação e Comunicação (TIC). Portanto, a EaD, particularmente no domínio do e-learning, configura-se como potencializador nos diversos níveis de formação (formal, não-formal e informal), visto que possibilita aliar as tecnologias e a aprendizagem de forma colaborativa por meio dos dispositivos móveis.

Os dispositivos móveis são a TIC mais onipresente e bem-sucedida da história da humanidade. Elas existem em grandes quantidades, em lugares onde livros e escolas são escassos. Em menos de uma década, as tecnologias móveis se espalharam para os lugares mais longínquos do planeta. Da população estimada da Terra, por volta de 7 bilhões de pessoas, 6 bilhões já têm acesso a um telefone móvel em funcionamento (UNESCO, 2014). Portanto, a tecnologia móvel tem mostrado como aliada e propulsora de uma educação inclusiva e de qualidade.

Este artigo apresenta o conceito de usabilidade e as suas articulações vinculando a usabilidade técnica e pedagógica na aprendizagem móvel e ubíqua. Expondo quais os possíveis benefícios da aprendizagem móvel e ubíqua e seus desafios. $\mathrm{O}$ artigo é um estudo inicial da investigação que enquadra-se na linha Development Research que se aplicam a variadíssimas situações de pesquisa educativa, em geral, e à investigação do domínio da Tecnologia Educativa (TE), em particular (Van den Akker, 1999). Trata-se de um modelo de investigação multi-metodológico, na medida em que permite ao investigador a possibilidade de combinar métodos quantitativos e qualitativos. Porquanto, a análise documental, a observação, estudo de caso, inquérito por questionário ou por entrevista e registros automáticos.

\section{Da educação a distância à educação sem distâncias}

A EaD no contexto de desenvolvimento e disseminação das TIC, mostra-se como mais uma possibilidade de socialização dos conhecimentos e expansão da educação (da Silva, Beche, Bock, \& Souza, 2012). Segundo Santarosa (2000), com a ampliação da Internet, expande-se as possibilidades de $\mathrm{EaD}$, não somente pelo acesso ao saber e à informação, mas, principalmente, porque fomenta a criação de alternativas metodológicas de intervenção pedagógica. Consequentemente, abre-se espaço de oportunidades, especialmente para as pessoas cujos padrões de aprendizagem não seguem os quadros típicos de desenvolvimento.

A EaD tem se fortalecido com o advento e avanço das TIC, para além das necessidades sociais e econômicas da sociedade. Daí a preocupação dos responsáveis pelas políticas educativas na Europa e no mundo em responderem a este apelo da internacionalização e atualização do ensino, tentando criar um amplo debate (Pacheco, 2003) sobre as necessidades que surgem na vida dos cidadãos a fim de desenvolver competências de aprendizado ao longo da vida.

Correlato as mudanças de comportamento ocasionados pela expansão das TIC na educação, os dispositivos móveis emergem como um aliado nos diversos níveis de 
formação educacional e objeto de pesquisas. Segundo a Organização das Nações Unidas para Educação, a Ciência e a Cultura (UNESCO, 2014) os dispositivos móveis podem ampliar e enriquecer as oportunidades educacionais para estudantes em diversos ambientes. As tecnologias móveis (smartphones, tablets, notebooks, entre outros) proporcionam um inovado ambiente de aprendizagem, que darão forma ao ensino-aprendizagem do século XXI. Essas tecnologias móveis alcançam seus usuários em qualquer lugar ou tempo, diminuindo as distâncias entre aprendiz e professor e aprendiz-aprendiz, anulando as barreiras, físicas, econômicas e sociais. Portanto, o e-learning assim como o m-learning proporciona novos meios de abordar e minimizar as deficiências do ensino tradicional e remover a distância.

\section{Aprendizagem Móvel (Mobile Learning)}

Há anos que o número de telemóveis e smartphones superou o número de computadores pessoais, convertendo-se no sistema de comunicação interpessoal por excelência, e atualmente utilizado com potencial alternativa na expansão do ensino.

O termo mais utilizado para nos referirmos aos estudos de investigação que relacionam a mobilidade, aprendizagem e tecnologias móveis é o mobile learning ou m-learning. Winters (2007) assinala que será muito difícil uma definição do m-learning, em razão de que é um campo de pesquisa relativamente recente (aproximadamente, um decênio), que ainda está em fase de evolução, o que torna difícil a existência de unanimidade sobre o termo (Caudill, 2007).

Para Batista, Behar, \& Passerino (2010), a conceitualização dada ao m-learning é ainda emergente e um pouco ambígua. Por isso, consideramos necessária mais perquirições para que se possa falar do m-learning como um "paradigma educacional" (Moura, 2011).

O tema aprendizagem móvel pode ter diferentes abordagens, assim como o desenvolvimento da tecnologia em si (do hardware e infraestrutura operacional), as teorias educacionais que dão sustentação à sua aplicação na aprendizagem em movimento, ou ainda as implicações sociais dessas práticas educacionais (Traxler, 2009). De acordo Certal \& Carvalho (2011) estudos apontam para uma perspectiva que vá ao encontro do usuário e da ubiquidade das comunicações pois no m-learning são diversas a natureza da aprendizagem (formal ou informal) dentro e fora da sala de aula e do conhecimento.

Há mais ou menos uma correlação com termos como wireless, ubiquidade, aprendizagem pervasiva ou nômade, porém nenhum desses termos sozinho representa plenamente o conceito de m-learning. Situações de divergências acerca de termos e conceitos são típicas de disciplinas emergentes.

Inicialmente o m-learning foi associado a mediatizar a aprendizagem pela utilização das tecnologias móveis (Batista et al., 2010). Assim, muitos dos investigadores focavam a definição deste novo conceito sob a perspectiva tecnológica, como uma aprendizagem que se realizava através da utilização de dispositivos móveis, entre as quais: PDA (Personal Digital Assistant), iPod, smartphone, playstation portable, notebook, tablet PC, reprodutores MP3 e telemóveis.

De acordo com a UNESCO (2014), aprendizagem pode ocorrer de várias formas, entre elas: as pessoas podem usar aparelhos móveis para acessar recursos educacionais, conectar-se a outros estudantes ou desenvolver conteúdos didáticos, dentro ou fora da sala de aula. A aprendizagem móvel também abrange esforços em apoio a metas educacionais amplas, tais como o ensino formal (presencial ou a distância), informal (cursos livres) e nos diversos níveis de ensino (do ensino básico a pós-graduação), como a administração eficaz de sistemas escolares e a melhor comunicação entre a escola e família.

O documento da UNESCO (2014) contém um guia com 10 recomendações para que governos implantem políticas públicas e utilizem telemóveis ou smartphones como recurso em sala de aula. Tais como:

1. Criar ou atualizar políticas ligadas ao aprendizado móvel.

2. Conscientizar sobre sua importância.

3. Expandir e melhorar opções de conexão.

4. Ter acesso igualitário.

5. Garantir equidade de gênero.

6. Criar e otimizar conteúdo educacional.

7. Treinar professores.

8. Capacitar educadores usando tecnologias móveis.

9. Promover o uso seguro, saudável e responsável de tecnologias móveis.

10. Usar tecnologia para melhorar a comunicação e a gestão educacional.

Entretanto, existem desafios no m-learning que necessitam ser superados tais como: requisitos de padronização de software educativo, conteúdo educacional adaptado aos diversos tipos de dispositivos móveis, desconhecimento do seu uso ou receio no ambiente de ensino e a usabilidade. A usabilidade no m-learning propicia que os usuários tenham facilidade de aprendizagem, eficiência e acessibilidade em seu uso.

\section{Da usabilidade técnica à usabilidade pedagógica}

A usabilidade pode ser definida como o grau em que um determinado campo do software auxilia alguém a realizar uma tarefa, sem tornar-se um obstáculo adicional para sua realização. Os objetivos dos sistemas de utilização são geralmente avaliados através de vários critérios tais como: facilidade de aprendizagem; retenção de aprendizagem ao longo do tempo; velocidade de conclusão da tarefa; taxa de erro e satisfação subjetiva do usuário (Nielsen, 1990). A usabilidade é considerada “o fator que assegura que os produtos sejam fáceis de usar, eficientes e agradáveis, da perspectiva do usuário" (Preece, Rogers, \& Sharp, 2005).

Usabilidade implica na otimização das interações estabelecidas pelas pessoas com produtos interativos, a fim de possibilitar que realizem suas atividades no trabalho, na escola ou em atividades de lazer, é dividida nas seguintes metas:

a) Eficiência: cumprir os objetivos para o qual foi criada; 
b) Segurança: permitir o manuseio de forma segura para o usuário e para o próprio sistema;

c) Utilidade: ser útil para o usuário e as atividades que ele pretende desempenhar;

d) Capacidade de aprendizagem: ser uma interface de fácil aprendizado para o usuário;

e) Capacidade de memorização: ser fácil de lembrar como se usa.

Além das metas de usabilidade, a expansão do uso das TIC pelo público em geral impulsionou diferentes metas decorrentes da experiência do usuário.

Os princípios de Interação Humano-Computador (IHC) além de pesquisar a usabilidade, incluem consistente foco em usuários finais, no caso dessa investigação refere-se aos professores e estudantes e em suas funções, medições empíricas do uso do sistema e desenvolvimento interativo. Muito esforço foi empenhado em explorar modelos cognitivos do comportamento humano referente ao uso do computador, e desenvolver diretrizes para layout de tela e diálogos com os sistemas .

A norma ISO 9241:11 (1998) prescreve que a medida da usabilidade para o software (denominada Usabilidade de Design) determina "a capacidade de um produto ser usado por usuários específicos para atingir objetivos específicos com eficácia, eficiência e satisfação em um contexto específico de uso."

No conceito da ISO 9241:11 (1998), os três pontos essenciais são:

Eficácia - Capacidade dos usuários conseguirem o que necessitam usando o produto. Para se medir a eficácia é comum verificar se os usuários concluíram a tarefa proposta.

Eficiência - Habilidade que requer do usuário a utilização do produto com menor esforço. Normalmente a medição é feita utilizando uma escala fixa para se calcular o tempo gasto pelo usuário para realizar determinada tarefa.

Satisfação - Está associada ao bom desempenho e disposição e contentamento no uso de determinado software. É possível identificar pelas atitudes físicas ou subjetivas do usuário na sua interação com o produto. Esta satisfação é externada pelas atitudes positivas ou não, quando o usuário responde ao questionário ou pela sua observação no momento em que ele realiza as tarefas.

Embora frequentemente os estudos de usabilidade concentra-se nos aspetos tecnológicos e de design de interface, um outro conceito começa a emergir no campo do uso das TIC, assim como nos ambientes de aprendizagem e que investigaremos neste artigo: o conceito de usabilidade pedagógica.

A usabilidade pedagógica é definida de acordo com a classificação de Nielsen (1990), como um sub-conceito de utilidade; e a usabilidade técnica como um sub-conceito de usabilidade conforme mostra a Figura 1. Deste modo, além do diálogo entre um usuário e um sistema, a usabilidade pedagógica de um sistema e/ou material de aprendizagem é também dependente de metas estabelecidas pelo estudante e pelo professor em uma situação de aprendizagem (Nokelainen, 2006).

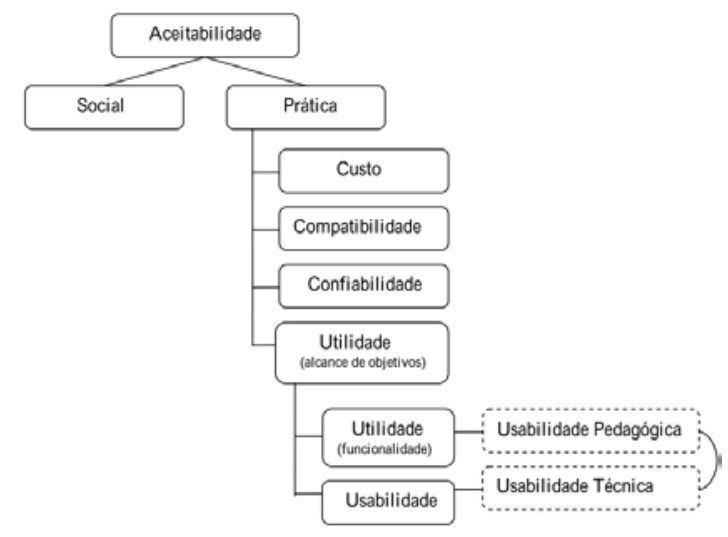

Figura 1.Mapeamento conceitual da usabilidade técnica e pedagógica pelo modelo de usabilidade de Nielsen. Fonte: Nielsen (1990) adaptado por Nokelainen (2006) apud Reitz (2009).

A Usabilidade Pedagógica (UP) refere-se à necessidade de aprendizagem significativa e à utilização de ambientes para aprendizagem construtivista. As características da aprendizagem e o uso da tecnologia são inter-relacionados, interativos e interdependentes (Martins, 2004).

A usabilidade pelo viés pedagógico poderá ser abordada através da construção do conhecimento, inserindo-o numa perspectiva construtivista, em que o sujeito participa ativamente na construção do seu saber, tornando-se, por isso mesmo, mais responsável pela sua aprendizagem.

Nesse sentido, Nokelainen (2006) estabeleceu dez critérios para mensurar a UP de materiais didáticos, com base em estudos empíricos que envolvem as dimensões: controle por parte do aprendiz, atividade do aprendiz, aprendizagem colaborativa, orientação aos objetivos, aplicabilidade, valor agregado, motivação, valor do conhecimento prévio, flexibilidade, e feedback.

Segundo Silius \& Tervakari (2003) avaliação da UP está relacionada com os objetivos pedagógicos elegidos e com o valor agregado esperado. Os objetivos pedagógicos são influenciados pelas metas de aprendizagem do conteúdo, assim como pelos métodos de ensino e de aprendizagem.

A UP prioriza o desenvolvimento de materiais centrados no aprendiz, por outro lado a usabilidade técnica privilegia as funcionalidades do sistema para o aprendiz utilizá-la. Porém o maior desafio da EaD é manter a motivação do aprendiz e envolve-lo em processos participativos, afetivos e interativos. Portanto, o material didático precisa ser autoexplicativo e elaborado de modo a permitir e facilitar ao aprendiz intervir e controlar o curso de sua aprendizagem. (Agner, 2009).

\section{Considerações finais}

Conforme asseverou-se inicialmente este artigo trata-se de tese em andamento cujo objetivo é articular os conceitos de Usabilidade Técnica e Usabilidade Pedagógica aplicados no âmbito da aprendizagem móvel e ubíqua. Portanto, não há dados substanciais para a 
formulação de quaisquer conclusões, pois situa-se em fase inicial. Todavia, é-nos possível afirmar que a expansão dos ambientes virtuais de aprendizagem, o elevado uso de tecnologias móveis e outros fatores têm promovido notáveis transformações junto a comunidade acadêmica de diversas áreas. Eis, portanto, a razão de nossa escolha teórica em diminuir o hiato entre a usabilidade técnica e usabilidade pedagógica no cenário contemporâneo. $\mathrm{O}$ que muito provavelmente aprimorará ensino, desde o letramento a pós-graduação. Fatores como: colaboração e envolvimento de usuários de sistemas educativos e tecnologias móveis e ubíquas; as preferências dos educadores (profissionais) em métodos pedagógicos potencializados pelas tecnologias educativas - tornar-se-ão soluções promissoras ao status quo na área da Educação.

\section{Referências}

Agner, L. (2009) Inovação e Qualidade do Design na Educação Online: Uma Contribuição à Usabilidade Pedagógica. ABED.

Batista, S. C. F., Behar, P. A., \& Passerino, L. M. (2010, December). Recursos pedagógicos para dispositivos móveis: uma análise com foco na matemática. RENOTE, 8(3), 10.

Caudill, J. G. (2007, June). The growth of m-learning and the growth of mobile computing: Parallel developments. International Review of Research in Open and Distance Learning, 8(2), 13.

Certal, F. M., \& Carvalho, A. A. (2011). Estudo sobre receptividade ao m-learning no ensino básico. VII Conferência Internacional de TIC na Educação, (pp. 1427 - 1438).

Da Silva, S. C., Beche, R. C. E., Bock, G. L. K., \& Souza, C. P. (2012). Educação Inclusiva E Educação A Distância : Contribuições Desenho Do Universal Para Aprendizagem Na Composição Do Moodle. Associação Brasileira de Educação a Distância, 1-10.

Lemos, A. (2007). Ciberespaço e Tecnologias Móveis. Processos de Territorialização e Desterritorialização na Cibercultura. In Sulina (Ed.), Imagem, visibilidade e cultura midiática.Livro da XV COMPÓS. Porto Alegre.

ISO 9241:11 Ergonomic requirements for office work with visual display terminal (VDTs. (1998). The internacional organization for standartization.

Martins, M. D. L. O. (2004). O Papel Da Usabilidade No Ensino A Distância Mediado Por Computador. Centro Federal De Educação Tecnológica De Minas Gerais.Dissertação de Mestrado.

Moura, A. M. C. (2011). Apropriação do telemóvel como ferramenta de mediação em mobile learning: estudos de caso em contexto educativo. Universidade do Minho.

Nielsen, J. (1990). Evaluating hypertext usability. Designing Hypermedia for Learning, pp. 147-168.

Nokelainen, P. (2006). An empirical assessment of pedagogical usability criteria for digital learning material with elementary school students. Educational Technology and Society, 9(2), 178-197.
Pacheco, J. A. (2003). Políticas educativas para o ensino superior na União Europeia: um olhar do lado português. Educação \& Sociedade, 24, 17-36.

Preece, J., Rogers, Y., \& Sharp, H. (2005). Design de Interação: Além da Interação Homem-Computador. (Bookman, Ed.). Porto Alegre: Trad. Viviane Possamai.

Reitz, D. S. (2009). Avaliação do impacto da usabilidade técnica e pedagógica no desempenho de aprendizes em e-learning.

Santarosa, L. M. (2000). Telemática y la inclusión virtual y social de personas con necesidades especiales : un espacio posible en la Internet. $\mathrm{V}$ Congresso Ibero-Americano de Informática Na Educação-RIBIE.

Silius, K.; Tervakari, A-M. 2003. The Usefulness of Web- based Learning Environments: The evaluation Tool into the Portal of Finnish Virtual University. International Conference on Network Universities and e-learning, Valencia, Spain.

Traxler, J. (2009). Current State of Mobile Learning. Mobile Learning: Transforming the Delivery of Education and Training (pp. 9-24). AU Press, Athabasca University.

UNESCO - Organização das Nações Unidas para a Educação a Ciência e a Tecnologia (2014). O Futura da Aprendizagem Móvel - Implicações para Planejadores e Gestores de Políticas.

UNESCO (2014). Diretrizes de políticas da UNESCO para a aprendizagem móvel. Paris.

Van den Akker, J. (1999). Principles and methods of development research. In Design approaches and tools in education and training. Springer, pp. 1-14. Netherlands.

Winters, N. (2007). What is mobile learning? Big issues in mobile learning: Report of a workshop by the kaleidoscope network of excellence mobile learning initiative. University of Nottingham.

\section{Agradecimentos}

Agradeço ao programa de cooperação internacional CAPES/COFECUB na Universidade do Minho. Financiado pela CAPES - Agência Federal de Apoio e Avaliação de Nível Superior do Ministério da Educação do Brasil. 